\title{
EL LENGUAJE ESCÉNICO DE LETIZIA RUSSO
}

SCENIC LANGUAGE OF LETIZIA RUSSO

Fausto Díaz Padilla

Universidad de Oviedo

\section{Resumen:}

Este artículo trata de analizar el teatro de Letizia Russo, dramaturga italiana del siglo XX, a partir de su obra Tomba di cani. Se hace un análisis de los elementos teatrales (personajes, tiempo, espacio, conflicto...), así como de la lingüística misma del texto (signos de puntuación, estructura del lenguaje, etc.).

\section{Palabras clave:}

Letizia Russo, lenguaje, signos de puntuación, análisis

\section{Abstract:}

This article tries to analyze the theater of Letizia Russo, an Italian playwright of the 20th century, based on her play Tomba di cani. An analysis is made of the theatrical elements (characters, time, space, conflict...), as well as the linguistics of the text itself (punctuation marks, language structure, etc.).

\section{KeYWORDS:}

Letizia Russo, language, punctuation marks, analysis 
Dramaturga Letizia Russo nació en Roma en 1980. En1998, siendo estudiante de enseñanza media, gana el premio “Grinzane Cavour-Grinzanescrittura" con Il dialogo fra Pulcinella e Gesù, que rehízo con posterioridad con el título Niente e nessuno para su estreno en el año 2000 en el Festival Per Antiche Vie del Teatro de Roma. En el año 2001 gana el premio Pier Vittorio Tondelli con Tomba di cani.

El título de la obra Tomba di cani posee un valor simbólico y hace referencia al lugar en el que se desarrolla una guerra lejana y próxima al mismo tiempo. Ese lugar se llama Cinossema, nombre formado por dos términos griegos que significan precisamente "tumba de perros" que da título a la obra. Es un lugar en el que mueren y son enterrados "los perros" de la guerra, en tal cantidad que los funerales se celebran de forma masiva e ininterrumpida. (Luther: cuando describe los funerales).

Pero el lugar en el que los personajes viven, mejor dicho, "representan" sus vidas, es también un lugar abstracto que puede ser cualquier lugar, ya que el objetivo de la autora es poner en escena una serie de sentimientos, vivencias, pensamientos del ser humano se encuentre donde se encuentre. Por esa razón en la ambientación de la Escena V se dice "In un non-luogo", acotación escénica que podría haber sido hecha ya en la Escena I.

Y lo mismo sucede con el concepto de tiempo. En efecto, el tiempo de la obra es un tiempo abstracto, un no-tiempo dado que, ni siquiera, existe una referencia a un tiempo simbólico como en el caso del lugar, de Cinossema.

Se trata, por tanto, de una no-concreción espacio-temporal pues de lo que se trata es de exponer la universalidad del alma humana ante conceptos intrínsecos y consustanciales con ella. Tales como su existencia en las vertientes más dramáticas cuales son el paso del tiempo, la muerte, la violencia, el odio, la guerra, etc.

La obra presenta una estructura de escenas-flash con pocos personajes. Escenas como piezas de un puzle que luego se irán combinando y que tendrán sentido en el conjunto o a medida que el cuadro del conjunto se va formando. En efecto, a través del diálogo se van esbozando cuadros sueltos de las vidas de sus protagonistas: de la relación de Johnny con su madre, con Mània, con su hermana, con su amigo Vin, con Luther; la de Glauce, la madre, con su hijo Johnny, con Mània y con Vin; la de éste con recuerdos de infancia -el asesinato de su hermano de 6 años y con su padre- con Johnny y con Glauce; la relación de Mània con Johnny, con Luther, con Glauce, con el Ejército, etc. Se trata, pues, de un entretejido de relaciones de unos personajes con otros, pero son relaciones puntuales entre sí que, quizá, queden difuminadas en el cuadro general de la obra y que en ningún caso crea caracteres verdaderos. Probablemente, ni siquiera, arquetipos de comportamientos. 
La acción, mejor dicho, los diálogos de la obra se hallan distribuidos en 14 escenas. En cada una no intervienen más de tres personajes lo que da idea de la concreción del tema que se desarrolla en cada uno. De acuerdo con su peso en la obra predominan las escenas breves. De un total de catorce, ocho ocupan un espacio de dos páginas; cuatro entre cuatro y cinco páginas y, sólo una, la escena central, o sea, la octava, se extiende por ocho páginas a pesar de que los personajes sean sólo dos. Por otro lado, la distinción de escenas no responde a la regla básica de distinción de los personajes que es la entrada o salida de un personaje. En algunas de ellas como la IV o la XIV entra un personaje -en la primera es Vin, y en la XIV Johnny- y no se produce transición a otra escena.

El número de personajes en cada escena es mínimo: entre dos y tres. De las catorce escenas sólo en cuatro los personajes son tres, en tanto que en las diez restantes los personajes son sólo dos: el número mínimo para que exista diálogo, la base del teatro. En total los personajes son seis. Sus edades son aproximadas, no precisas y oscilan entre los 25-30 años a los 70-75. Las dos únicas mujeres presentan las edades extremas: la más joven, Mània, entre 25-30, y la mayor, Glauce, entre 70-75. En medio, entre 30-35 años los dos hombres que se disputan el amor de la mujer joven. El amigo-enemigo de uno de ellos, Vin, es un poco mayor entre 35-40 años. Parece como si la autora rindiera homenaje a Dante, quien consideraba que la mitad "del camino de la vida" eran los 35 años, situando el final en los 70. Los dos personajes más abstractos -Alto grado del ejército, Tercer hombre- tienen la misma edad, entre 55-60 años, hasta el punto de que pueden ser interpretados por el mismo actor, según acota la propia autora.

Los nombres de los personajes con reminiscencias eslavas trasladan mentalmente al espectador a una hipotética nación del este de Europa.

El destino de la obra teatral no es la lectura privada, sino la representación sobre un escenario ante un determinado número de espectadores por lo que se busca, sobre todo, el efecto escénico. Este destino determina que el texto de una obra de teatro sea un elemento más, sin duda el más importante, del lenguaje escénico. En efecto, el libreto de la obra forma, junto con otros elementos (como la interpretación -o sea, la recitade los actores, la dirección de la obra, la decoración del escenario, la luminotecnia, la tramoya, etc.) el lenguaje escénico. Tan importantes son dichos elementos en el acto de la representación que pueden hacer triunfar o fracasar una obra. No es raro oír o leer el juicio crítico sobre una obra teatral en el que se opina que su fracaso se debe a una mala interpretación de los actores o que no han estado a la altura del texto. $\mathrm{O}$ a la inversa, que un texto mediocre sea "salvado" por la brillante interpretación de los actores.

Es ésta la perspectiva desde la que se ha de enjuiciar el libreto de la obra Letizia RUSSO Tomba di cani. Su lenguaje -pues no se puede hablar de un "estilo" propio, característico- es breve, conciso, telegráfico a veces. La misma grafía aparece incompleta 
en lo relativo a los signos de puntuación -comas, puntos, interrogación, exclamación, etc.- ya que debe ser el actor quien ha de dar la entonación justa, adecuada a la escena y al momento de la representación siguiendo las directrices interpretativas marcadas por el director. Es decir, es como si la autora dejara "incompleta" la obra para que sea completada en el escenario por los agentes de la representación.

Simplicidad del lenguaje escénico: Como se ha dicho, la escritura escénica de Letizia RUSSO es breve, concisa, en la que predomina lo que se quiere transmitir más que el modo cómo transmitirlo. O sea, el predominio del fondo sobre la forma. De ahí que predominen las estructuras gramaticales simples lo que se refleja en parlamentos relativamente breves. Las más frecuentes son las estructuras verbales, los indicadores de la acción para dar órdenes, consejos, sugerencias, etc. con el ánimo de intervenir sobre la voluntad del interlocutor:

JOHNNY Ascolta. Te lo devi prendere. Mi devi un favore. Io non te lo volevo ricordare. Mi ci hai costretto tu. Mi devi un favore, non ti puoi rifiutare. Non lo puoi fare per un anello del cazzo che ci guadagni più di me (p. 22)

Parlamento que se inicia con un verbo el imperativo, le sigue una perífrasis de obligación, continuando después con unas explicaciones justificativas de sus órdenes o peticiones precedentes.

Si bien en el lenguaje escénico de la RUSSO predominan las estructuras verbales, también son frecuentes las estructuras nominales, en particular las preguntas o respuestas con un sustantivo, un pronombre, un adjetivo o un adverbio. Incluso una combinación de varios de esos elementos en parlamentos brevísimos:

MÀNIA Be' insomma. Quelli sono. Storie.

A veces se trata de tres personajes distintos, o sea el máximo de personajes en una misma escena, con un parlamento de un solo término cada uno:

$3^{\circ}$ UOMO Che favore

JOHNNY (ride) Che bastardo

VIN (ride davvero) 'Stardo.

Lenguaje breve, impreciso, telegráfico.

Cuando el parlamento de un personaje es más largo de lo habitual se debe a que posee valores de narración de algo sucedido.

El análisis del lenguaje escénico, que no estilo, de L. RUSSO se va a centrar en los signos de puntuación, o sea, en su ausencia; en la sintaxis de la frase compuesta y de la frase simple, y en aspectos morfológicos de algunos elementos gramaticales como los pronombres personales y los demostrativos. 


\section{LOS SIGNOS DE PUNTUACIÓN}

Si algo caracteriza el modo de escribir de la dramaturga es la escasez o la ausencia de signos de puntuación. Escasez de puntos, de comas y ausencia absoluta de todos los demás: de signos de interrogación, de exclamación, de comillas cuando se reproduce palabras de un tercer personaje, etc.

Donde mejor se aprecia la falta de signos de puntuación es en aquellos parlamentos relativamente largos en los que se narra o se imagina un hecho ya ocurrido o que puede tener lugar de un momento a otro, pues se trata de un acontecimiento reiterativo de la existencia humana, como es el parto de una criatura. Así es como aparece en el texto, sin signos de puntuación para indicar las pausas:

MÀNIA ... Lei va in ospedale saluta il maritino saluta pure la macchinina così per tenerezza e dice alla macchinina Vedrai la prossima volta saremo in tre. Un altro bacino al maritino ciao scompare dietro la porta dell'ospedale. Naturalmente c'è la pace tutto è al suo posto i medici gli infermieri neanche macchia per terra (p. 31).

En la siguiente reproducción, con una separación y un guión bajo se indican las pausas que se deberían hacer:

MÀNIA ... Lei va in ospedale_ saluta il maritino _ saluta pure la macchinina _ così per tenerezza e dice alla macchinina _ Vedrai la prossima volta saremo in tre. Un altro bacino al maritino _ ciao_scompare dietro la porta dell'ospedale. Naturalmente _ cè la pace _ tutto è al suo posto _ i medici _ gli infermieri _ neanche macchia per terra (p. 31).

En otras ocasiones no se trata de la descripción de un hecho reiterativo, atemporal, sino de algo concreto, puntual, en el que las preguntas y las respuestas se suceden pero tienen en común con el parlamento anterior que quien se interroga y se responde es el mismo personaje aunque sea en nombre de otros:

La domanda giusta è__Ci manderete al fronte _ E la risposta è_Sì__naturalmente. Ma la vera domanda che mi aspetto da voi è__ Come volete che combattiamo._E per rispondere a questo vi dirò: premieremo i migliori. Premieremo chi avrà iniciativa. Chi avrà fantasia nell'iniziativa. $O$ chi verrà ferito. E premieremo anche chi morirà (p. 24)

En donde se aprecia una cierta anarquía en la puntuación, pues en la segunda parte de este parlamento los signos de puntuación, los puntos y seguidos concretamente, aparecen usados de modo correcto cuando se podría, incluso, prescindir de ellos, si esa parte se considerase una enumeración.

Con relativa frecuencia la falta de signos de puntuación puede dar lugar a diversas formas de interpretación que el director de la obra y el actor han de resolver: 
MÀNIA Non è vero non le dicevo quelle cose non mi piacciono gli uomini non avevo niente contro di lei.

En donde es de suponer las pausas después de vero, de cose y de uomini.

MÀNIA Non è vero _ non le dicevo quelle cose non mi piacciono gli uomini non avevo niente contro di lei.

Sin embargo, si no se hiciera pausa después de cose este vocablo sería considerado sujeto de mi piacciono y el sentido de la frase cambiaría totalmente: quelle cose non mi piacciono.

A veces la pausa, aunque no indicada gráficamente, resulta evidente por otros indicios grficos. Tal sucede con el adverbio de negación que en italiano adopta dos formas: no ante pausa, y non ante forma verbal. Esta peculiaridad se evidencia con claridad en el siguiente diálogo:

MÀNIA No. No grazie ... (p. 19)

En donde la pausa tras primera negación aparece reflejada con el punto, no así la segunda. Pero los indicios de que hay una pausa después del segundo no son: que no es la forma non y que se encuentra ante sustantivo pues la variante non va antepuesta a verbo. Por ello si la negación se halla ante verbo la indicación de la pausa puede resultar más dificultosa de detectar, como en el caso siguiente:

JOHNNY (non è molto in sé -per la droga-) Che cazzo sei un ascetta.

LUTHER No è la roba (p. 63)

Si esta última frase se recitase toda seguida tendría el significado contrario al que tiene, pues estaría negando la propiedad o el mal que se le atribuye a "la roba", mientras que el personaje lo que está haciendo es afirmarlo:

LUTHER No. È la roba.

Esa anarquía en el uso de los signos de puntuación, y en concreto de la interrogación, resulta evidente en aquellos casos en que se suceden dos preguntas sucesivas realizadas por el mismo personaje y dirigidas al mismo interlocutor: en la primera pregunta se hace caso omiso del signo de interrogación y en la segunda aparece:

JOHNNY Qual è stata la prima cosa che ti sei dimenticata.

E la seconda? (p. 42)

Quizá la ausencia de signo de interrogación en la primera pregunta se halle justificada para la autora porque va introducida por el pronombre interrogativo qual, en tanto que la segunda lo hace directamente. En otras ocasiones sucede lo mismo:

E io poi che ci faccio (p. 22) 
Es decir, la presencia de una marca interrogativa, un pronombre interrogativo, hace que la autora considere como no necesario el signo de interrogación.

Asimismo la autora hace omisión de los signos de exclamaciónevidentes, en particular cuando hay expresiones en imperativo:

VIN Lascia stare dai ti dico che quello là il mio amico se ci parlo... (p. 22)

En donde la puntuación sería:

VIN Lascia stare! Dai! ti dico che quello là il mio amico. Se ci parlo...

Y como éste otros muchos casos, incluso en la misma escena.

También ausencia de signos de puntuación, y en particular de exclamaciones, se aprecia en el siguiente parlamento al igual que en otros muchos:

UOMO No guarda lascia stare.

La puntuación para guiar al actor en la declamación sería:

UOMO No. Guarda! lascia stare!

O sea, punto después de la negación; punto o exclamación después de guarda, y signo de exclamación después de la perífrasis obligativa lascia stare.

Respecto a la grafía y, quizá, por un estúpido ateísmo militante de la autora, escribe con minúsculas las referencias a nombres de personajes de la religión cristiana como Cristo o la Virgen:

GLAUCE Abbiamo sempre pregato a cristo, noi. E alla madonna (p. 40)

O las referencias a la misma divinidad:

LUTHER Una volta ho parlato con dio lo sai. (p. 63)

Podría argumentarse que, como se trata de un texto para ser declamado sobre un escenario y no para ser leído, la falta de mayúscula en los nombres propios religiosos carece de importancia. Pero esta justificación no es válida, pues las referencias a nombres propios de otros personajes aparecen con mayúsculas.

Ya se ha aludido al hecho de que la escritura teatral, por hallarse destinada a su declamación en un escenario y no a la lectura privada, posee unas características particulares relativas a los signos de puntuación, a las grafías de los nombres propios, etc. Características que persiguen una mayor libertad interpretativa por parte del director, de los actores y de cuantos participan en el montaje de la obra.

En lo que se refiere al lenguaje, al texto de la obra, la libertad en su uso, que en ocasiones se convierte en anarquía, lleva a que sea un lenguaje directo, inmediato, en el que prima la expresión del pensamiento o la intencionalidad de los personajes sobre cualquier finalidad o motivación artística. Esa finalidad determina las características 
del lenguaje: distorsión sintáctica cuando el parlamento del personaje es relativamente amplio, un lenguaje nominal o verbal de pocos términos, que va a lo esencial, que refleja las peculiaridades del habla media del italiano, como la práctica desaparición del subjuntivo. Pero, incluso en los parlamentos más breves la ruptura sintáctica también se produce entre frase/oración principal y subordinada/dependiente, entre los elementos de un tiempo compuesto, entre el sustantivo y sus determinantes, etc.

\section{LA ESTRUCTURA DEL LENGUAJE}

En el periodo: Los casos de distorsión sintáctica en el período resultan relativamente frecuentes en los pocos parlamentos amplios que hay en Tomba di cani. Como ejemplo, véase el siguiente caso:

MÀNIA Intendo tutto quanto che ti pago la libertà. Che se lo sa che io che sono una femmina te la pago a te che sei un uomo crepa (pág. 36)

Parlamento que leído pierde parte de su significado pero que, en la representación escénica, con la entonación y las pausas adecuadas, con la gesticulación de la actriz y la del actor su interlocutor y el resto de los elementos del escenario y de la representación escénica alcanza su completo significado.

MÀNIA Intendo tutto quanto che ti pago la libertà. Che se lo sa_ che io _ che sono una femmina _te la pago a te__ che sei un uomo _ crepa (pág. 36)

En algún caso la distorsión sintáctica, unida a la ausencia de signos ortográficos, hace que resulte difícil, o cuando menos ambigua, la interpretación de las palabras del personaje:

GLAUCE È che a pensare non me ne importa più niente di nessuno. Se mi mettevo a pensare prima capace che certe cose non le facevo. Capace che ci vedevo ancora.

Para comprender en todo su significado estas palabras de la madre ciega resulta imprescindible verla y escucharla en la representación escénica.

En la frase compuesta: Es a nivel de frase en donde las dislocaciones y rupturas sintácticas son más frecuentes. Son habituales en las frases completivas entre el verbo de la principal y la frase introducida por che

GLAUCE ... Mi ricordo. Che piangevi. (p. 20)

En el presente caso la ruptura es entre el verbo y su complemento directo, función que desempeña la proposición completiva.

$\mathrm{O}$, incluso, la ruptura del che completivo con el elemento del que depende se produce también cuando éste no es un verbo principal como en el caso precedente sino un adverbio: 
VIN Adesso che. Lavoro. Lo so che cosa è una strada dritta (p. 68)

y la ruptura se produce entre el che y el verbo que debería introducir. O sea, en el caso anterior la ruptura era a izquierda, y en éste lo es a derecha.

Otras veces la ruptura sintáctica se produce entre una proposición de relativo y su antecedente. Así, en el parlamento anterior aparece el siguiente caso:

MÀNIA Sì. Voglio dire no_non proprio. Non è un rumore. È come un rumore. Che ti prende in giro (p. 20)

Las separaciones de las frases dependientes -completivas o de relativo- de sus correspondientes principales las usa la dramaturga para marcar las pausas escénicas. Pausas que podría señalarlas con puntos suspensivos y la estructura gramatical se mantendría. En los casos citados sería:

Mi ricordo ... che piangevi (completiva)

E adesso che ... lavoro

En un caso la pausa se realiza antes del che, en el otro después, según se pretenda resaltar uno u otro elemento.

È come un rumore ... che ti prende (relativa)

Se trata, pues, de un lenguaje escénico que busca el efecto escénico, no la estructura gramatical.

En la frase simple: A nivel de frase simple se ha de diferenciar, simplemente por razones de estructuración, entre el sintagma verbal y el nominal.

a) En el sintagma verbal, dos son los aspectos más característicos de la escritura escénica de Letizia RUSSO.

El primero refleja el modo de hablar de la sociedad italiana actual, sobre todo de los más jóvenes, en lo relativo al uso del subjuntivo. Mejor dicho, al no uso incluso en casos en que sería necesario emplearlo como aconseja la gramática. Como, por ejemplo, después de una estructura de essere + adjetivo:

MÀNIA Allora è inutile che te lo racconto (p. 21)

O dependiendo de un verbo que indica voluntad, como volere:

GLAUCE ... O vuoi che seguo la pista dell'odore (p. 26)

O cuando depende de una estructura impersonal:

GLAUCE ... Basta che mi dici dov'è che lo metti (p. 26) 
La casuística podría prolongarse a todos aquellas estructuras sintácticas que requieren subjuntivo pero que en la lengua actual no se emplea, lo que ha llevado a teorizar sobre la muerte del subjuntivo.

Un segundo aspecto a destacar en el sintagma verbal, y éste es propio del lenguaje escénico de la autora, es la ruptura o dislocación entre los elementos de un tiempo compuesto, o sea, entre el auxiliar y su participio, para marcar la pausa escénica. Así, por ejemplo:

JOHNNY Mi sto vendendo di tutto /.../ Lo sai lo sapevi ieri notte sono. Disperato. Vatti a fare una passeggiata. (p. 25)

donde contrasta la escasez de signos de puntuación con la separación por un punto entre los elementos del passato prossimo. Lo mismo sucede en el siguiente caso entre otros varios:

VIN Sì che è un santuario. Tra qualche anno lo sarà. In memoria di tutta la gente che ci ha. Lavorato (p. 69)

El punto entre auxiliar y el participio del tiempo compuesto viene a indicar la pausa, más o menos larga, que debe guardar el actor. Lo sorprendente es que el participio se escriba con mayscula pues, aunque va después del punto, la autora no guarda mucho respeto a los signos ortográficos y menos a las mayúsculas.

b) En el sintagma nominal: Otro aspecto gramatical en el que parece regir cierta anarquía es en el empleo de los pronombres personales. Unas veces parece responder a un uso descuidado de los pronombres como el que se hace en el lenguaje coloquial. Así, la no correspondencia

JOHNNY ... E siamo soli io e per tutto il paradiso (p. 50)

en donde, por mantener esa correspondencia casual de los pronombres, debería ser o bien ambas formas en caso complemento:

Siamo soli me e te

o bien, ambas en forma sujeto:

Siamo soli io e tu

Quizá, lo que ha podido pretender la autora es destacar la relevancia del personaje que habla como elemento predominante de la escena.

La alternancia de pronombres se da incluso referido a la primera persona: uno bajo la forma sujeto y otro bajo la forma átona de complemento:

MÀNIA È che a qualcuno gli tocca che i figli glieli spara un cecchino. Io mi è toccato che gliela levo io la vita. Se guardi bene gli faccio pure un favore a farlo finire io (p. 31) 
en donde entre la abundancia de pronombres personales predomina la reiteración del pronombre sujeto de primera persona io. Pero en la segunda frase de este parlamento se suceden el pronombre sujeto io y el pronombre complemento átono mi, para concluir la frase con io:

Io mi è toccato che gliela levo io la vita.

Se trata de una frase circular pues se inicia y se concluye con io, pues la vita que va a continuación iría después de pausa - de una coma o un punto- ya que es especificación del pronombre la del pronombre combinado gliela. Sin lugar a dudas en la representación escénica entre el primer io y el mi que le sigue el actor debe guardar una pausa más o menos larga, pues es como si iniciara una frase con el io ... frase que queda abortada por otra introducida por el mi:

Io ... mi è toccato che gliela levo io.

La reiteración, a principio y final de la frase, del pronombre io marca su centralidad, su importancia y, a través de ella, el remordimiento y el intento de justificación del comportamiento del personaje.

También persigue el mismo objetivo de resaltar, de situar en primer término, el pronombre sujeto alejándolo del verbo y separándolo por pausa del resto de la frase:

GLAUCE ... I miei genitori andavano nelle chiese cristiane. Abbiamo sempre pregato a Cristo, noi (p. 40)

Si el pronombre noi apareciera delante del verbo carecería de relevancia ya que va implícito en los morfemas de persona y número del verbo $-1^{\underline{a}}$ de plural- $\mathrm{y}$, en consecuencia, podría omitirse sin que la frase perdiera valor significativo:

(Noi) abbiamo sempre pregato a Cristo

Más incorrecto resulta el uso del pronombre complemento indirecto masculino gli para referirse a una mujer:

GLAUCE Tua sorella era inteligente.

JOHNNY ... Tu la crescevi ma la odiavi. La odiavi. Cercavi di fargli male. Di fargli credere che avere marito e figli e pulire la casa era la cosa più bella. Tu lo sapevi che non era cosí... (p. 41)

Parlamento en el que contrasta el uso del pronombre complemento directo femenino la con el complemento indirecto masculino gli referido al mismo personaje, a la hermana de Johnny.

Frecuente en la lengua coloquial es el empleo de los pronombres demostrativos, en particular del genérico quello/ -a para referirse a personas no presentes en el diálogo o 
a sucesos acaecidos más o menos alejados en el tiempo. Baste como ejemplo la siguiente sucesión de parlamentos entre Johnny y Vin:

JOHNNY Quello è perchè fanno schifo che non se li lava mai (sentido neutro "eso")

VIN Lei è quella enorme che raccoglie il riso ... (femenino "aquella")

JOHNNY E quello ci sbava dietro (masculino: y "ese")

VIN Dice che il padre a quello gli ha fatto vedere la pistola (masculino: a "ese")

JOHNNY L'ha minacciato

JOHNNY E quello gli ha detto di no. (masculino)

VIN Mentre se ne andava il padre di quella ... (femenina)

JOHNNY E a quello come gli è venuta di fargli vedere la pistola. (masculino)

VIN Parlavano. Quello gli diceva le solite stronzate. (masculino)

Si se es escritor no tanto por lo que se dice sino por el cómo se dice, se ha de concluir que Letizia RUSSO no es una escritora. Es una autora teatral, una dramaturga, de mayor o menor éxito que se ha de circunscribir al escenario y al mundo del teatro, pero no al de la literatura en general. Y no es escritora porque en un somero análisis de su lengua -pues no se puede hablar de estilo- se aprecia que se trata de una lengua simple, de escasos recursos sintácticos, en la que predominan los elementos nominales o verbales de manera aislada. Y en la que, de manera voluntaria, hasta los aspectos gráficos aparecen descuidados: la mayoría de las veces por defecto; otras por exceso como el caso de la puntuación entre los elementos de dos frases -como entre el che completivo y el verbo regente, o el che relativo y su antecedente- o los elementos de una misma frase, como entre el auxiliar y el participio en los tiempos compuestos o el incorrecto uso de los pronombres personales.

\section{REFERENCIAS BIBLIOGRÁFICAS}

Fratus, Tiziano, "Cinque atti d'un teatro della materia e della morte per Letizia Russo", Editoria spettacolo, publicado el 19 de abril de 2004, en www.editoriaespettacolo.it Fratus, Tiziano, "Mentre Sarah Kane. Tre voci nella recente drammaturgia italiana", Manifattura, en www.manifatturae.it, 2004.

Fratus, Tiziano, "Pù̀ lamore sopravvivere all'olocausto?", antes en Drammaturgie en el portal www.dramma.it, Roma, 2002, después en L'architettura dei fari: 19902003, la nuova drammaturgia italiana, Borgomanero, Edizioni Atelier, 2003.

Fratus, Tiziano, “Tomba di cani”, Manifatturae, en www.manifatturae.it, 2002.

Garrone, Nico, "Il Nuovo Teatro. Travolti dalla carica dei ventenni sesso, disperazione e tanta ironia", La Repubblica, 28 de enero de 2003. 
Graham-Adriani, Suzy, "Binario Morto al National Theatre”, in Intercity Connections. Nuovi testi per nuovi interpreti, por Rodolfo di Giammarco y Barbara Nativi, Roma, Editoria \& Spettacolo, 2004.

Groppali, Enrico, "Tomba di cani, cruda poesia di un mondo maledetto", Il Giornale, 1 de mayo de 2003.

Maragnani, Laura, "Siamo noi la meglio gioventù: teatrale", Panorama, 18 de septiembre de 2003.

Martín Clavijo, Milagro, "Il teatro delle miserie di Letizia Russo: dialogismo en 'Tomba di cani'"', Escritoras y pensadoras europeas, Sevilla, Archivel Editores, 2007, pp.461483.

Nativi, Barbara, "Intercity Connections: il progetto", in Intercity Connections. Nuovi testi per nuovi interpreti, por Rodolfo di Giammarco y Barbara Nativi, Roma, Editoria \& Spettacolo, 2004.

Quadri, Franco, “Quando Fassbinder tentò un film su Auschwitz”, La Repubblica , 2 de octubre de 2002.

Russo, Letizia, “Chi cosa dove come quando perché", Matità - rivista online di drammaturgia geopolitica, ManifatturAE (www.manifatturae.it), Torino, mayo de 2003.

Russo, Letizia, “Diario di lavoro", en Russo, Letizia, Tomba di cani, Pistoia, Associazione Teatrale Pistoiese / Teatro del Tempo Presente, 2003. 